\title{
Metabolic syndrome and diabetes mellitus in women with and without stress urinary incontinence
}

\author{
Jan Brož ${ }^{1}$ (D) Michaela Hronová ${ }^{1}$. Ludmila Brunerová ${ }^{2}$ \\ Received: 8 February 2019 / Accepted: 13 March 2019 / Published online: 23 March 2019 \\ (C) The International Urogynecological Association 2019
}

Dear Editor,

We read with interest the article by Ströher et al. entitled "Metabolic syndrome in women with and without stress urinary incontinence" published recently in your journal [1]. It presents the data from a valuable study, trying to compare the frequency of metabolic syndrome in patients with and without stress urinary incontinence. The main conclusion of the study was that metabolic syndrome frequency was higher in patients with stress urinary incontinence, suggesting a possible association between these two conditions.

The article provides a comprehensive list of studies related to the topic and engages in a profound discussion about the collected and analyzed data, leading to a very interesting debate.

Nevertheless, we would like to ask the authors one question that may contribute to further, more detailed discussion of the issue.

The authors correctly mentioned type 2 diabetes mellitus as a risk factor for stress urinary incontinence [2]. The definitions of metabolic syndrome which were used in the study $[3,4]$ are both based partly on the presence of elevated fasting plasma glucose or antidiabetic treatment, either of which may indicate diabetes mellitus. Unfortunately, the authors did not state if there were any diabetic patients among the study group. If there were, this could partially put the study results in doubt, suggesting that it could be the investigation of the relation of stress urinary incontinence not only to metabolic syndrome but at the same time also to diabetes mellitus (we could even speculate that all the patients diagnosed with metabolic syndrome could suffer from type 2 diabetes).

Jan Brož

zorb@seznam.cz

1 Department of Internal Medicine, Second Faculty of Medicine, Charles University, V Úvalu 84, 15000 Prague, Czech Republic

2 Second Department of Internal Medicine, Center for Research of Diabetes, Metabolism and Nutrition, Third Faculty of Medicine, Charles University, University Hospital Královské Vinohrady, Ruská 97, 10600 Prague, Czech Republic
Therefore, we would like to respectfully ask if the authors could provide information about the prevalence of diabetes among the study group, and we suggest taking into account this comment when deciding on the protocol of this interesting and important study if its continuation is planned.

Acknowledgments Special thanks to Dion Pritchard for language editing.

Funding This article was supported by the Ministry of Health, Czech Republic-conceptual development of research organization, Motol University Hospital, Prague, Czech Republic 00064203.

\section{Compliance with ethical standards}

This article does not contain any studies with human participants or animals performed by any of the authors.

Conflicts of interest None.

\section{References}

1. Ströher RLM, Sartori MGF, Takano CC, de Araújo MP, Girão MJBC. Metabolic syndrome in women with and without stress urinary incontinence. Int Urogynecol J. 2019. https://doi.org/10.1007/ s00192-019-03880-6.

2. Brown JS, Vittinghoff E, Lin F, Nyberg LM, Kusek JW, Kanaya AM. Prevalence and risk factors for urinary incontinence in women with type 2 diabetes and impaired fasting glucose. Diabetes Care. 2006;29:1307-12.

3. Third Report of the National Cholesterol Education Program (NCEP). Expert panel on detection, evaluation, and treatment of high cholesterol in adults (adult treatment panel III)- final report. Circulation. 2002;106(25):3143-421.

4. International Diabetes Federation: the IDF consensus world-wide definition of the metabolic syndrome. 2005. http://www.idf.org/ webdata/docs/Metac_def.pdf. Accessed 02 Feb 2019.

Publisher's note Springer Nature remains neutral with regard to jurisdictional claims in published maps and institutional affiliations. 